\title{
Mycobacteriophage D29 contains an integration system similar to that of the temperate mycobacteriophage $\mathbf{L 5}$
}

\author{
Graça Ribeiro, ${ }^{1} \dagger$ Miguel Viveiros, ${ }^{2}$ H. L. David ${ }^{2}$ and João V. Costa ${ }^{1} \dagger$ \\ Author for correspondence: João V. Costa. Tel: +351 13632141 . Fax: + 35113632105. \\ e-mail: jvcosta@ihmt.unl.pt
}

1 Laboratory of Molecular Virology, Gulbenkian Institute of Science, Apartado 14, P-2781 Oeiras Codex, Portugal

2 Department of Microbiology and Centre of Studies of Malaria and Other Tropical Diseases, Institute of Hygiene and Tropical Medicine, R. Junqueira 96, 1300 Lisboa, Portugal

\begin{abstract}
A mycobacteriophage D29 DNA fragment cloned in pRM64, a shuttle plasmid that transforms Mycobacterium smegmatis, was sequenced. The determined sequence was 2592 nucleotides long and had a mean G+C content of $63.7 \mathrm{~mol} \%$, similar to that of mycobacterial DNA. Four ORFs were identified: one with strong homology to dCMP deaminase genes; one homologous to mycobacteriophage L5 gene 36, whose function is unknown; one encoding a possible excisase; and one encoding an integrase. The intergenic region between the putative excisase gene and the integrase gene had a lower than average $G+C$ content and showed the presence of the same attP core sequence as mycobacteriophage L5. Transformation experiments using subclones of pRM64 indicated that the integrase gene and all the intergenic region were essential for stable transformation. A subclone containing the integrase gene and the core attP sequence was able to transform but recombinants were highly unstable. Southern analysis of total DNA from cells transformed with pRM64 and its derivatives showed that all the plasmids were integrated at one specific site of the bacterial chromosome. A recombinant exhibiting a high level of resistance to the selective drug kanamycin had two plasmids integrated at different sites. These results demonstrated that the D29 sequences contained in PRM64 were integrative, indicating that the generally held view of D29 as a virulent phage must be reviewed.
\end{abstract}

Keywords: Mycobacterium, mycobacteriophage D29, integrase, site-specific integration, dCMP deaminase

\section{INTRODUCTION}

Mycobacterial diseases, namely tuberculosis and leprosy, still remain important world-wide public health problems. This has been seriously aggravated during the last decade by the increase of drug-resistant tuberculosis affecting AIDS patients (Snider et al., 1994). The understanding of pathogenesis and drug resistance, the search for new therapeutic drugs, and the preparation of a new generation of vaccines require further advances in the present knowledge of the molecular biology of mycobacteria. This strongly depends on the availability of efficient tools for genetic analysis and manipulation

+Present address: Laboratory of Molecular Biology, Institute of Hygiene and Tropical Medicine, R. Junqueira 96, 1300 Lisboa, Portugal.

The GenBank accession number for the sequence reported in this paper is U81553. of mycobacteria. The majority of shuttle vectors used in molecular biology of mycobacteria (Snapper et al., 1988; Lazraq et al., 1990; Stover et al., 1991; Barletta et al., 1992) derive from a Mycobacterium fortuitum plasmid, pAL5000 (Labidi et al., 1985). Because vectors containing the origin of pAL5000 are not always stable and transform inefficiently (Snapper et al., 1988; Lazraq et al., 1990), Lazraq et al. (1991) reasoned that the origin of replication (oriR) of a broad-spectrum virulent mycobacteriophage could be an alternative to pAL5000 in constructing stable multicopy plasmids. For this purpose, a plasmid (pRM64) was constructed containing the kanamycin-resistance gene from $\operatorname{Tn} 903$ and an origin, oriR, present in a Pst I insert from mycobacteriophage D29 (Froman et al., 1954).

Mycobacteriophage D29 has been extensively studied (reviewed by Mizuguchi, 1984). It is apparently related to another well-known mycobacteriophage, L5, because 
lysogens for L5 show immunity to superinfection with D29 (Donnely-Wu et al., 1993). However, there is an important difference between the two phages, i.e. L5 is a temperate phage (Lee et al., 1991) whereas D29 has been traditionally considered as a virulent phage (White et al., 1962).

Soon after the construction of pRM64, David et al. (1992) reported the preparation of a similar plasmid, pBL415. The sequence of this second plasmid has not been published but was deposited in the GenBank database (accession number X70352). The observation that this sequence contained an integrase-like gene was unexpected, considering that D29 is a virulent bacteriophage. Therefore, we thought that it was necessary to examine further the D29 fragment cloned into pRM64. In this paper, we report the full sequence of the mycobacteriophage D29 region inserted in plasmid pRM64 and the characterization of the minimal regions required for stable transformation of mycobacteria. We have also identified an ORF with strong homology to dCMP deaminases and three other ORFs, one encoding an integrase with strong similarity to L5 integrase and another encoding a putative excisase.

\section{METHODS}

Bacterial strains and media. Escherichia coli XL1-Blue (Stratagene) was grown in Luria-Bertani (LB) medium. When necessary, the medium was supplemented with $100 \mu \mathrm{g}$ ampicillin $\mathrm{ml}^{-1}$. The high-frequency transformation strain $\mathrm{Myco}$ bacterium smegmatis $\mathrm{mc}^{2} 155$ (Snapper et al., 1990) was cultivated in liquid medium RV10b (Redmond \& Ward, 1966) or on LB agar. Resistance to kanamycin was assayed by adding the desired concentration of the drug to the medium.

DNA extraction. Plasmid DNA was extracted from E. coli using a Qiagen plasmid midi kit, as recommended by the manufacturer. For minipreparation of $M$. smegmatis total DNA, cells were incubated until the late-exponential phase of growth and a loopful of cells was suspended in $500 \mu 10 \mathrm{mM}$ Tris/ $\mathrm{HCl}(\mathrm{pH} 8 \cdot 0), 1 \mathrm{mM}$ EDTA (TE), heated at $80^{\circ} \mathrm{C}$ for $20 \mathrm{~min}$ and treated with $1 \mathrm{mg}$ lysozyme $\mathrm{ml}^{-1}$ for $1 \mathrm{~h}$ at $37^{\circ} \mathrm{C}$. After $10 \mathrm{~min}$ incubation at $65^{\circ} \mathrm{C}$ with $100 \mu \mathrm{g}$ proteinase $\mathrm{K}$ $\mathrm{ml}^{-1}$ and $1 \%$ SDS, $\mathrm{NaCl}$ and cetyltrimethylammonium bromide were added to final concentrations of $0.7 \mathrm{M}$ and $33 \mathrm{mM}$, respectively. The mixture was incubated at $65^{\circ} \mathrm{C}$ for $10 \mathrm{~min}$ and DNA was extracted with chloroform/isoamyl alcohol, precipitated with cold ethanol, dried under vacuum, and dissolved in a small volume of TE.

DNA manipulations and hybridization. All DNA manipulations, restriction enzyme digestions, agarose gel electrophoresis and Southern hybridizations were performed following standard procedures (Sambrook et al., 1989).

Bacterial transformation. Competent $E$. coli cells were transformed by a classical calcium chloride technique (Sambrook $e t$ al., 1989). M. smegmatis was transformed by electroporation, as follows. A $100 \mathrm{ml}$ culture was grown to $\mathrm{OD}_{650} 0 \cdot 6$, cooled on ice for $30 \mathrm{~min}$ and centrifuged at $6500 \mathrm{~g}$ for $15 \mathrm{~min}$ at $4{ }^{\circ} \mathrm{C}$. The pellet was washed with cold water and then with cold $10 \%(\mathrm{v} / \mathrm{v})$ glycerol, and finally resuspended in a small volume of $10 \%$ glycerol, to obtain a final concentration of about $10^{9}$ c.f.u. $\mathrm{ml}^{-1}$. This cell suspension was distributed in small volumes and stored at $-70^{\circ} \mathrm{C}$. A $40 \mu \mathrm{l}$ aliquot was thawed, transferred to an electroporation cuvette and mixed with $1 \mu \mathrm{l}$ DNA solution containing 100 ng DNA. The cells were cooled on ice for $10 \mathrm{~min}$ and subjected to a $4.5 \mathrm{~ms}$ pulse using a Gene Pulser electroporator (Bio-Rad) set at $1.25 \mu \mathrm{F}, 200 \Omega, 25 \mathrm{kV}$ $\mathrm{cm}^{-1}$. Cells were immediately diluted in $1 \mathrm{ml} \mathrm{RV10b}$ liquid medium and incubated at $37^{\circ} \mathrm{C}$ for $3 \mathrm{~h}$ under mild agitation. They were then spread on LB agar plates containing $10 \mu \mathrm{g}$ kanamycin $\mathrm{ml}^{-1}$.

DNA sequencing. pRM64 and the subclones containing pRM64 BamHI or SalI subfragments were sequenced by the dideoxy chain-termination method (Sanger et al., 1977). Both strands were completely sequenced. For sequencing, $1 \mathrm{pmol}$ of double-stranded plasmid DNA was denatured in $0.2 \mathrm{M} \mathrm{NaOH}$ and $2 \mathrm{mM}$ EDTA, neutralized and precipitated with ethanol. The denatured DNA was used as template for sequencing, using a Sequenase (version 2.0) kit from United States Biochemical as recommended by the manufacturer. Sequencing primers were standard primers corresponding to both flanking regions of the vector DNA or specific primers synthesized on the basis of sequence already read. To confirm the sequence of regions with GC stretches resulting in band compression, dITP was used in some experiments instead of dGTP. Sequencing was also performed by cycle reactions using Taq DNA polymerase and fluorescent-dye-coupled dideoxy terminators. The products were analysed with a 373A automated DNA sequencer (Applied Biosystems). Analysis of DNA and protein sequences was performed with the programs of the University of Wisconsin Genetics Computer Group (Devereux et al., 1984).

Assay of plasmid stability. Three independent M. smegmatis transformants from each plasmid were inoculated into RV10b medium and grown to saturation. Cultures were diluted $1: 100$ in medium without kanamycin and grown again to saturation. Additional passages were done for a total of 160 generations. At $3 \mathrm{~d}$ intervals, aliquots of the same volume were plated on solid medium with or without $10 \mu \mathrm{g}$ kanamycin $\mathrm{ml}^{-1}$ and the percentage of drug-resistant cells was calculated $4 \mathrm{~d}$ later.

\section{RESULTS}

\section{Nucleotide sequence of the mycobacteriophage D29 integration region}

We determined the sequence of the 2592 nucleotide mycobacteriophage D29 PstI insert in pRM64. The phage insert had a high $\mathrm{G}+\mathrm{C}$ content $(63.7 \mathrm{~mol} \%)$ typical of mycobacterial DNA. However, there was a central region of about 600 nucleotides showing a moderately lower $\mathrm{G}+\mathrm{C}$ content of $60 \cdot 2 \mathrm{~mol} \%$. Direct or inverted repeats of significant length were not detected. The sequence showed several possible ORFs on both strands. We considered four of these ORFs as putative genes because their codons corresponded to frequently used codons in a table compiled from known mycobacterial and mycobacteriophage genes and because they were preceded by consensus ribosomebinding sequences (Kempsell et al., 1992). The positions and orientation of these four ORFs, designated g1, g2, g3 and g4, are indicated in Fig. 1. The D29 insert comprises only the 3 -part of ORF g1, which starts in an adjacent DNA fragment. All the ORFs were oriented in the same direction. The first three genes mapped closely 
(a)
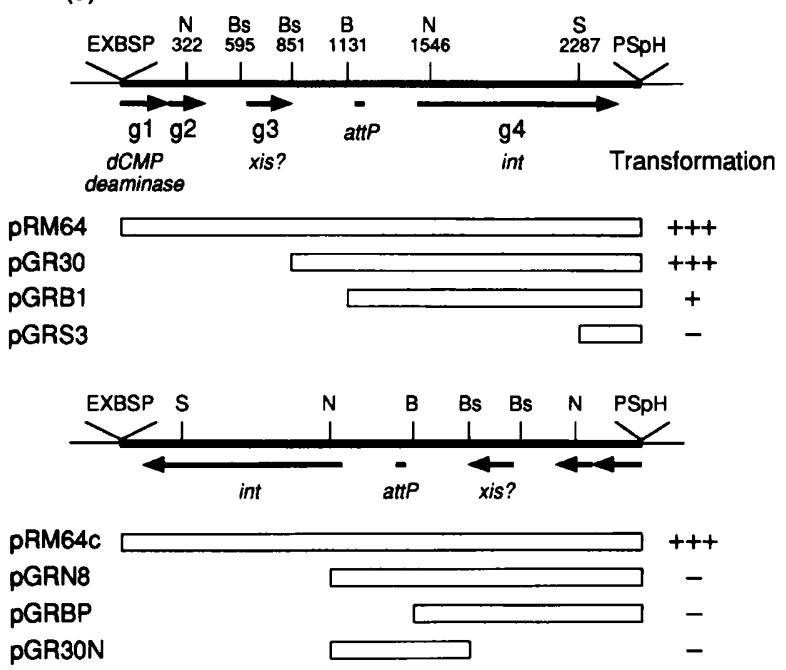

(b)

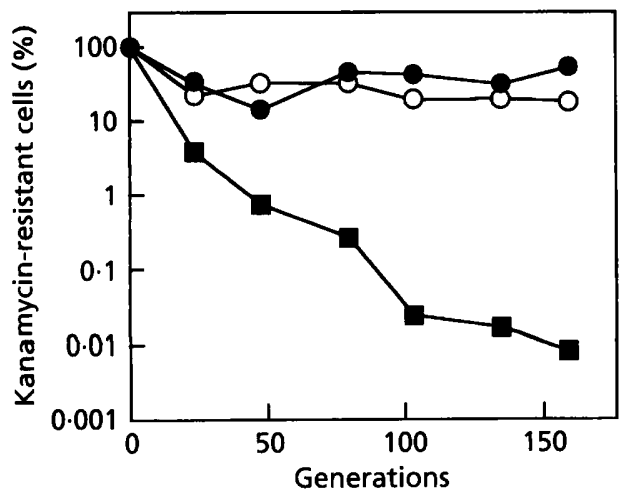

Fig. 1. (a) Representation of the DNA fragment from mycobacteriophage D29 cloned in pRM64 as well as of the subcloned derivatives described in the text. Restriction enzyme sites: B, BamHI; Bs, BstXI; E, EcoRI; H, HindIII; N, Nael; P, Pstl; S, Sall; Sp, Sphl; X, Xbal. (b) Plasmid loss under non-selective conditions from cells transformed with pRM64 (O), pGR30 (O) and PGRB1 $(\mathbb{C})$. The experiments were done in triplicate with consistent results. The generation time of $M$. smegmatis under our experimental conditions was estimated to be $3 \mathrm{~h}$.

in the left third of the sequence but the fourth gene was separated from the others by a large intergenic region of 620 nucleotides, corresponding to the region with lower $\mathrm{G}+\mathrm{C}$ content. The two left-most genes were contiguous, with the termination codon of $\mathrm{g} 1$ immediately upstream of the ATG codon of g2. Therefore, the putative translation start signal of $\mathrm{g} 2$ was part of the coding sequence of $\mathrm{g} 1$.

We could only assign a probable function to three of the genes, as described below. The other putative gene, $\mathrm{g} 2$, encodes a small protein of 56 amino acids, with a predicted $M_{\mathrm{r}}$ of 6322 . This hypothetical protein showed $89 \%$ similarity to the product of mycobacteriophage L5 gene 36 (Hatfull \& Sarkis, 1993), whose function is unknown.

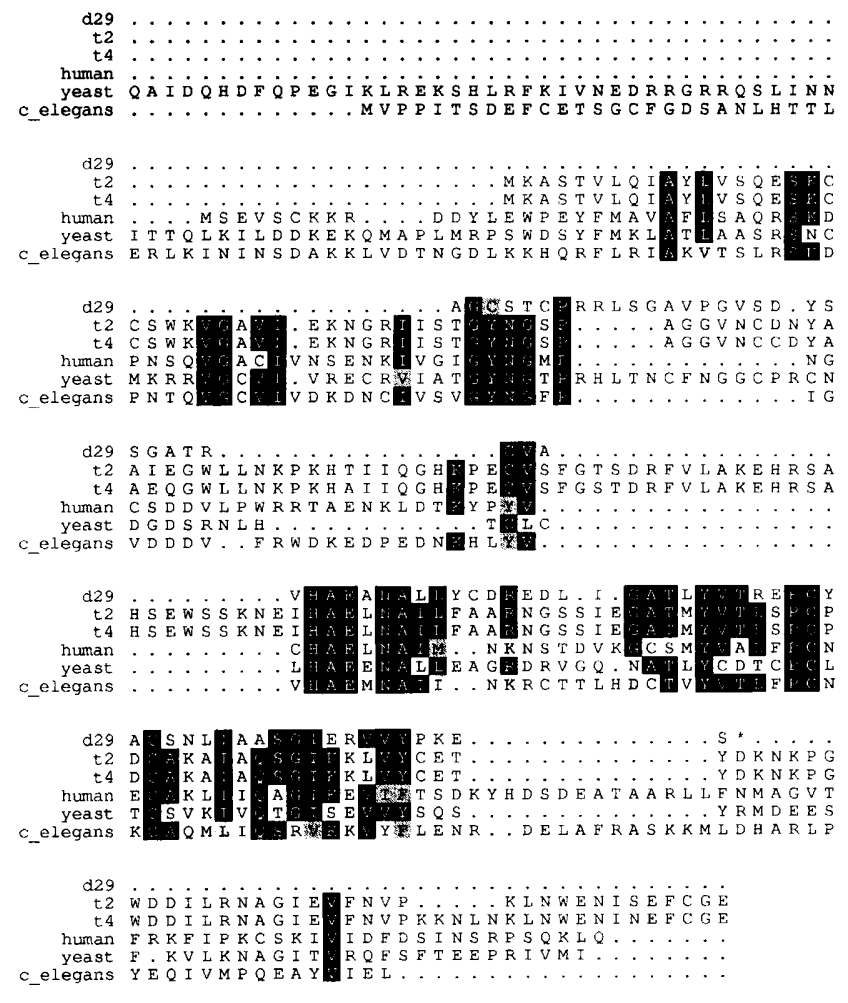

Fig. 2. Multiple alignment of the predicted product of ORF $g 1$ to dCMP deaminases from Caenorhabditis elegans, Homo sapiens, bacteriophages T2 and T4, and Saccharomyces cerevisiae (SWISS-PROT accession numbers P30648, P32321, P00814, P16006 and P06773, respectively). Perfect matches are indicated by black boxes and conservative substitutions by grey boxes. The first 100 amino acids of the yeast enzyme were omitted from this alignment.

\section{D29 probably has a dCMP deaminase gene}

A FASTA search of the deduced product of $\mathrm{g} 1$, the leftmost ORF of the D29 insert, revealed a strong homology to dCMP deaminase genes. Fig. 2 shows a multiple alignment of dCMP deaminases (EC 3 .5 .4 .12) from bacteriophages $\mathrm{T} 2$ and $\mathrm{T} 4$, Saccharomyces cerevisiae, Homo sapiens, Caenorhabditis elegans and the predicted product of the D29 11 gene. These proteins share two conserved central domains separated by a spacer of variable length and flanked by amino and carboxyl-terminal tails that have little or no homology. The partial D29 gene sequence found in the pRM64 insert comprised only part of the left conserved domain, which was contiguous to the other conserved domain. In this sense, the D29 gene was more related to the eukaryotic genes than to the $\mathrm{T}$-even genes.

\section{The D29 genome encodes an integrase and possibly an excisase}

The region of the D29 insert in pRM64 showing the highest level of similarity to mycobacteriophage L 5 was the $3^{\prime}$-half of the sequence, covering gene 44 in D29 and gene 33 in L5. This gene encodes an integrase (Lee et al., 


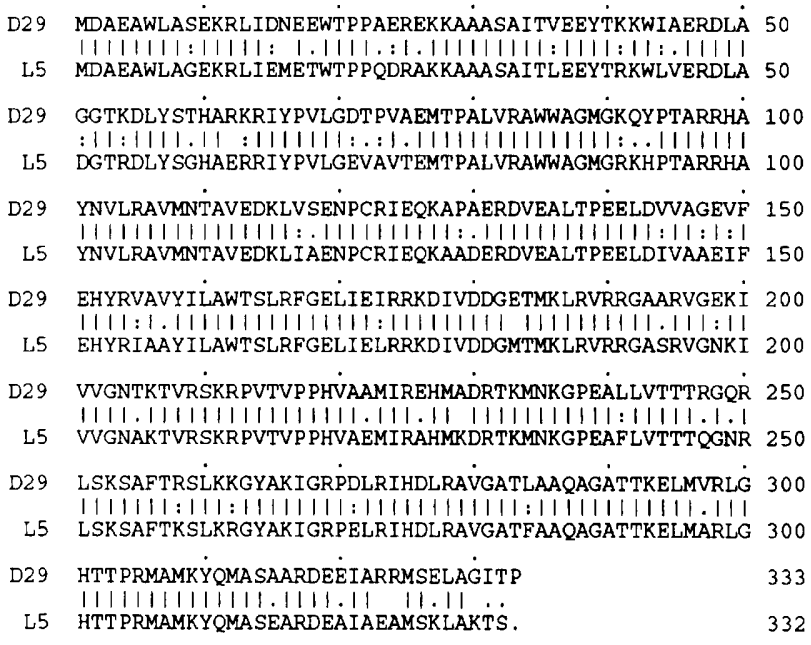

Fig. 3. Alignment of the amino acid sequence of the putative D29 integrase with the sequence of mycobacteriophage L5 integrase. Vertical bars indicate perfect homology and colons or dots indicate conservative substitutions.

1991; Hatfull \& Sarkis, 1993; Lee \& Hatfull, 1993). Correspondingly, the predicted product of D29 gene g4, 333 amino acids long, had a high degree of similarity $(83.5 \%)$ to the L5 integrase (Fig. 3). Most of the sequence divergence was due to conservative substitutions.

Comparison of the DNA sequences in this region of both bacteriophages also disclosed the presence in D29 of the same attP core sequence as in mycobacteriophage L5 (Lee et al., 1991; Hatfull \& Sarkis, 1993). The 42 nucleotide attP core sequence mapped in the lower $\mathrm{G}+\mathrm{C}$ content region and was oriented to the right (Fig. $1)$.

After identifying the integrase gene, we considered the hypothesis that D29 also encodes an excisase gene. The presence of L5 DNA to the right of the attP site confers instability to plasmids, which led to the suggestion that an excisase gene, probably gene 34 , is present in that region (Lee et al., 1991; Hatfull \& Sarkis, 1993). Like L5 gene 34, the D29 ORF g3 is adjacent to the attP site at its $5^{\prime}$-side and corresponds to that L5 gene. Comparison of the D29 ORF g3 and L5 gene 34 revealed $60 \%$ similarity. Therefore, $\mathrm{g} 3$ might be a candidate excisase gene. FASTA searches using $\mathrm{g} 3$ or its predicted product as query sequences did not result in significant homology to any known protein. As an alternative approach, we performed a multiple alignment of the deposited sequences of excisases from coliphages lambda, P2, P22, HK022 and $\phi 80$, from Staphylococcus phage L54a, from transposon Tn1545 from Streptococcus pneumoniae, and from Saccharopolyspora erythraea. From this multiple alignment no conserved motifs were detected, but a homology profile was extracted using program ProfileMaker. We compared this profile to the predicted product of gene g3, using the program ProfileGap, and observed a functional homology of $61 \%$. However, in the absence of experimental evidence, the role of $\mathrm{g} 3 \mathrm{as}$ a putative excisase is conjectural.

\section{Functional dissection of the D29 integration region}

If those two genes actually encode an integrase and an excisase, it would be interesting to know their role in the replication and stability of vectors based on the D29 integration region. To elucidate this issue, we prepared a set of subclones from pRM64 spanning different subregions of the original D29 insert (Fig. 1a).

Deletions in the 5 -region of the insert were prepared by digestion of pRM64 with BamHI and re-ligating to generate pGRB1, or by double digestion with BstXI and $X b a I$, treating with $\mathrm{S} 1$ nuclease and Klenow enzyme to generate blunt ends and re-ligating to generate pGR30. Both recombinants contain the putative integrase gene and lack the possible excisase gene. Clone pGR30 contains all the intergenic region, whereas pGRB1 contains only the attP sequence, without its upstream region. A much shorter recombinant, pGRS3, was constructed by excision of the Sall fragment of pRM64. To prepare deletions in the other end of pRM64, we constructed $\mathrm{pRM} 64 \mathrm{c}$, which contained the same Pst I fragment from D29 but in the opposite orientation. From this recombinant, we constructed subclone pGRBP by digestion with $B a m \mathrm{HI}$ and re-ligating. A shorter deletion was prepared by digestion of pRM64c with EcoRI and Nael, repairing the ends and blunt end ligation, generating pGRN8. Subclone pGRBP contained the putative excisase gene and the $5^{\prime}$-end of the intergenic region but lacked the attP sequence and the integrase gene. The other subclone, pGRN8, lacked only the integrase gene. Finally, we constructed a recombinant, pGR30N, lacking both genes but retaining all the intergenic region. To construct this recombinant, we first excised the BstXI-SphI fragment of pRM64c and then digested the resulting recombinant with EcoRI and $\mathrm{Nael}$. The protruding ends were filled by treatment with Klenow enzyme and re-ligated.

Recombinant pGR30 transformed $M$. smegmatis as efficiently as pRM64 or pRM64c, yielding about $10^{5}$ kanamycin-resistant transformants ( $\mu \mathrm{g}$ plasmid DNA $)^{-1}$. Recombinant pGRB1 was much less efficient, yielding only 600 transformants $(\mu \mathrm{g} \text { DNA })^{-1}$. No kanamycin-resistant transformants were observed after transformation with any of the other plasmids.

Subclone pGR30 was quite stable, as demonstrated by determination of the proportion of cells that had conserved kanamycin resistance (Fig. 1b). After 160 generations of non-selective growth, $53 \%$ of descendants from pGR30-transformed cells still maintained the plasmid. The original plasmid pRM64 was less stable, since only $17 \cdot 4 \%$ of descendants were resistant to kanamycin. Recombinant pGRB1, which was also able to transform $M$. smegmatis, although much less efficiently, was very unstable. The proportion of kanamycin-resistant descendants decreased progressively with cultivation and was lower than $0.01 \%$ when 
(a)

(b)

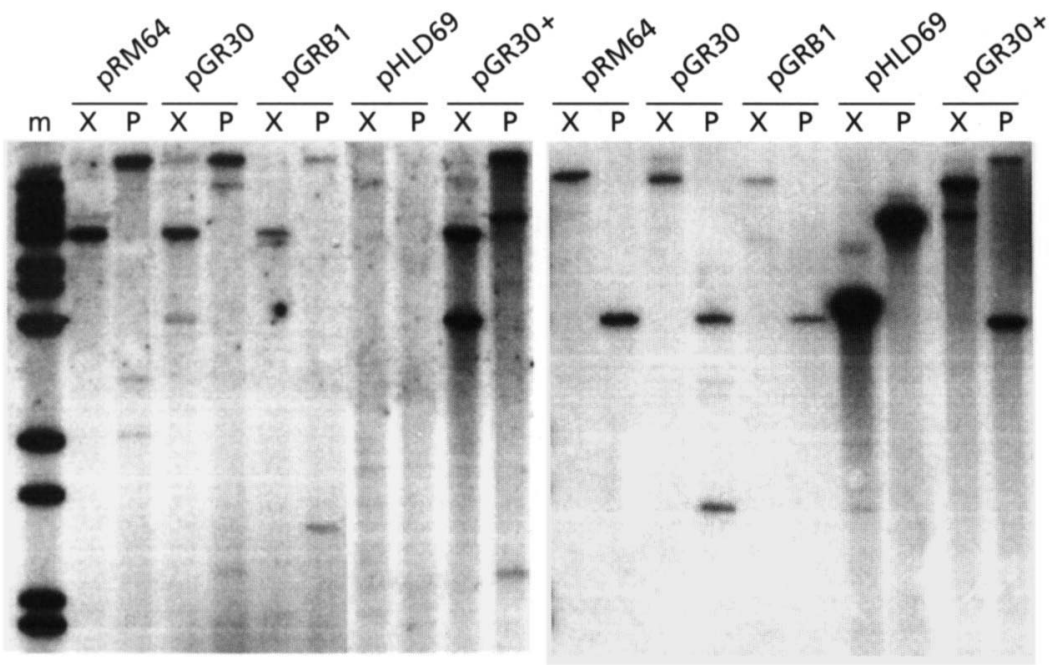

Fig. 4. Hybridization of cellular DNA from $M$. smegmatis transformed with PRM64, PGR30, pGRB1 or pHLD69 and from pGR30transformed cells showing a high level of resistance to kanamycin (pGR30+) with probes P1 (a) and P2 (b). DNA was digested with $X m n l(X)$ or Pvull $(P)$. Lane $m$ is a BstEll digest of lambda labelled DNA (dimensions in $\mathrm{kb}: 8.4,7 \cdot 2,6 \cdot 4,5 \cdot 7,4.8,4 \cdot 3,3.7,2.3,1.9$, 1.4 and 1.3 ). the culture reached 160 generations. No spontaneous kanamycin-resistant clone was observed in control cultures of non-transformed cells.

Taken together, these results indicated that the integrase gene and the complete intergenic region around the attP sequence were required for efficient transformation and for stability of plasmids based on the D29 origin. In addition, the removal of the putative excisase gene seemed to confer a higher degree of plasmid stability in successive cell generations.

\section{Chromosomal integration of D29-derived plasmids}

The presence of an integrase gene and its role in transformation and plasmid stability suggest that plasmids containing this D29 region may be integrated in the bacterial chromosome. To test this hypothesis, we performed a Southern analysis of pRM64 and its derivatives in transformed cells.

Total DNA from transformed cells was extracted and digested with $X m n \mathrm{I}$ or PvuII. XmnI cuts pRM64 once, in the pUC19 sequence, and there are two PvuII sites in pRM64, also in the pUC19 sequence. The digests were separated by agarose gel electrophoresis and hybridized with two different probes. One probe, $\mathrm{P} 1$, was the $1.5 \mathrm{~kb}$ BamHI-PstI fragment of pRM64 containing the integrase gene. The other probe, $\mathrm{P} 2$, was a $1.6 \mathrm{~kb}$ $\mathrm{XmnI-AatII}$ fragment of pRM64 containing the kanamycin-resistance gene and the $5^{\prime}$-region of the amp gene. Both fragments were electroeluted from gels and labelled.

As shown in Fig. 4, probe P1 revealed a single XmnI band, of $6 \mathrm{~kb}$, and a single PvuII band, of $11 \mathrm{~kb}$, in DNA from cells transformed with all tested plasmids, $\mathrm{pRM} 64$, pGR30 and pGRB1. A faint $X m n \mathrm{I}$ band of $3.7 \mathrm{~kb}$ and another faint $P v u I$ band of $8.9 \mathrm{~kb}$ were also detected in the pGR30 sample. The XmnI and PvuII bands detected in plasmid DNA extracted from E. coli had different sizes from those described above and corresponded to the sizes deduced from the plasmid sequences. Fragments with sizes larger than those expected from plasmid DNA were also detected when transformed cell DNA was hybridized with the P2 probe. In all tested DNAs, except those containing pHLD69, this probe detected a single $X m n \mathrm{I}$ band of $9 \cdot 4 \mathrm{~kb}$ and a PvuII band of $3.6 \mathrm{~kb}$. As expected, the PvuII band had the size of the larger PvuII plasmid fragment, since this enzyme cuts the plasmid twice, but the $X m n I$ band had a much larger size than the plasmid. In contrast to these results, two bands in each enzyme digest were detected when we assayed DNA from a cell culture derived from a pGR30 recombinant resistant to high doses of kanamycin, up to $1000 \mu \mathrm{g} \mathrm{ml}^{-1}$. Besides the bands described above, an equally strong $X m n \mathrm{I}$ band of $3.5 \mathrm{~kb}$ and a PvuII band of $6.4 \mathrm{~kb}$ were detected with the P1 probe. Probe P2 also detected additional bands of 6.6 and $10 \mathrm{~kb}$ in digests of $X m n I$ and PvuII, respectively. As control we used DNA from cells transformed with the autonomous plasmid pHLD69 (Houssaini-Iraqui et al., 1992), containing the pAL5000 origin. As expected, pHLD69 was not detected by probe P1. Probe P2 detected single XmnI and PvuII bands with the sizes expected for unintegrated plasmid bands.

\section{DISCUSSION}

Mycobacteriophages have provided replication origins or integration systems for the construction of vectors for genetic manipulation of mycobacteria (Lazraq et al., 1991; Lee et al., 1991; Stover et al., 1991; David et al., 1992). Mycobacteriophages may also provide active promoters driving the expression of foreign genes in recombinant bacille Calmette-Guérin. The insert that we sequenced, pRM64, is a plasmid shuttle vector containing a DNA fragment from mycobacteriophage D29 that was originally considered to be an origin of 
replication (Lazraq et al., 1991). A similar vector, pBL415, has also been described (David et al., 1992). The sequence of the D29 fragment inserted in this second vector was deposited in the GenBank database (accession number X70352) but to our knowledge it has not been published. Some differences in the two determined sequences result in considerable changes in the translation maps deduced from the sequences, mainly in the $5^{\prime}$-region of the fragment. Significant differences were found at position 175 in ORF g1, resulting in the introduction of a stop codon and a frameshift in the pBL415 sequence, and at position 664 in ORF g3, where the pBL415 sequence shows the absence of two nucleotides in a GC compression region. This explains why the authors did not describe the dCMP deaminase gene and the putative excisase gene that we have identified.

The dCMP deaminase gene is a salvage pathway gene which is also found in T-even bacteriophages (Hall \& Tessman, 1966; Maley et al., 1983) but not in lambdoid phages. Comparison of the putative D29 gene with known dCMP deaminase genes showed that the gene in the D29 insert is almost complete, probably lacking only the first 36 codons. The dCMP deaminase gene has not been identified in the D29-related mycobacteriophage L5, whose complete sequence is known (Hatfull \& Sarkis, 1993). Both phages show considerable similarity in a region corresponding to L5 gene 36 but the region immediately upstream, where the dCMP deaminase gene maps, shows high divergence between the two viruses.

A gene closely related to the L5 integrase gene (Lee \& Hatfull, 1993) was also identified. The D29 gene is functioning, since transformed $M$. smegmatis are very stable recombinants containing a single-copy insert of the plasmid, as demonstrated by Southern analysis of transformed cell DNA using two probes mapping diametrically opposed in the plasmid. If the plasmids were integrated, it would be expected that the probes would hybridize to different DNA fragments of cell DNA joined to plasmid sequences to each side of the attP sequence. In contrast, hybridization of autonomous plasmid DNA would only detect the corresponding plasmid sequence. Our results indicate that pRM64 and derivatives are integrated in the cell chromosome and we were not able to detect even trace amounts of free plasmid in the hybridization experiments. Transformation and plasmid integration were not observed when pRM64 derivatives without the integrase gene were tested. Single-copy vector integration may result in low levels of foreign gene expression but has the important advantage of ensuring a high stability of recombinants. This is critically required in situations where it is not possible to select directly for maintenance of the foreign gene or where selection by drug resistance is undesirable, as in the case of recombinant vaccines.

Plasmid pBL415, which is apparently identical to pRM64, is present in multiple copies in transformed cells (David et al., 1992). We do not have a plausible explanation for the discrepancy between these results and our data. This issue is difficult to discuss because the data on the copy number of pBL415 are not presented in that article.

In accordance with the integrase function, D29 also has an attP core sequence identical to that described for L5 (Lee et al., 1991), with the exception of the last nucleotide. The homology between L5 DNA and $M$. smegmatis DNA at this terminal position is probably only coincidental. However, the core sequence is not sufficient for efficient viral DNA integration and stability, as shown by the rapid plasmid loss by cells transformed with pGRB1. This plasmid has the core attP sequence but lacks the intergenic region upstream from the core sequence. All the D29 sequence $5^{\prime}$ of this intergenic region is not essential for stable transformation. This allows the construction of vectors like pGR30, with a much smaller insert than pRM64.

Our results on the dissection of the attP region agree with the recently published characterization of the mycobacteriophage L5 attP site (Peña et al., 1997). The L5 attP core sequence is flanked on both sides by armtype sites, designated P1-P7. Sites P1-P3, located between the int gene and the attP core sequence, and site P4, at the other side of the core sequence, are essential for transformation. Peña et al. (1997) compared the sequences of the attP region of L5 and D29 and found similar arm-type sites in D29. The sequence from the left end of the D29 insert in pRM64 to the BamHI site that was excised in pGRB1 contains the arm-type sites P4-P7. Therefore, at least site P4 is essential for efficient transformation, as in L5.

The attB site partially overlaps a tRNA ${ }^{\text {gly }}$ gene (Lee $e t$ al., 1991) and the points of strand exchange are located at the anticodon loop of the tRNA gene at attB (Peña et al., 1996). Therefore, integration is probably highly specific. The faint bands detected in Southern blots of DNA derived from transformed cells may correspond to alternative integration sites. Since the exact copy number of tRNA genes in M. smegmatis is not known, it is possible that other copies of attB provide alternative integration sites. In the same way, the high level of kanamycin resistance of a pGR30-transformed clone can be explained by integration and expression of two plasmid copies, as indicated by the hybridization results. The sizes of the additional bands detected in this experiment were not compatible with tandem integration in the same site, either in the same or in the inverted orientation. The integration within a tRNA gene can also explain the broad host range of mycobacteriophage D29, because tRNA genes are highly conserved. This may also indicate that D29 integrase can function in other mycobacterial species, thus enabling the construction of vectors that can be used for genetic manipulation of other species besides $M$. smegmatis. This possibility is currently being tested.

Hatfull \& Sarkis (1993) suggested that L5 gene 34, located $3^{\prime}$ from the att $P$ region, encodes an excisase, but experimental evidence for this assignment is still lacking. This gene corresponds to the g3 ORF that we identified 
in D29. As compared to D29, the L5 putative excisase gene is truncated at its $3^{\prime}$-end and extends further to the 5 -side. This makes the D29 homologue even more related to the homology profile of phage excisases than the L5 gene. If the g3 ORF actually encodes an excisase, pRM64 derivatives with this gene deleted will be expected to show increased stability. Although pGR30 was apparently more stable than pRM64, the difference in stability was not striking. Therefore, it is possible that D29 does not encode a functional excisase. Alternatively, excision may require accessory virus-induced functions coded by genes mapping in other D29 DNA fragments. Curiously, the most unstable of the plasmids that we have constructed was pGRB1, which lacks the putative excisase gene. A plasmid containing the integration region of mycobacteriophage L5 but lacking the arm-type sites P4-P7, like pGRB1, also showed low transformation efficiency and high instability (Peña et al., 1997). It is possible that the absence of those sites favours an excisase-independent excision, perhaps mediated by the integrase or by a cell factor.

Unlike L5, which is a temperate phage, D29 has been traditionally considered as a lytic phage (Froman et al., 1954; Bowman, 1958; Mizuguchi, 1984; Donnely-Wu et al., 1993). Different plaque morphologies were observed within the original isolate of D29 but this was due to differences in adsorption (Bowman, 1958) and lysogeny could not be demonstrated in infected colonies (White $e t$ al., 1962). Consistent with these facts and in the absence of sequencing data, the D29 fragment cloned in shuttle vectors pRM64 and pBL415 was previously considered to be an origin of replication (Lazraq et al., 1991; David et al., 1992). Our results clearly indicate that this is not the case and that the cloned sequence represents an integration region. Site-specific integration is a key feature of temperate phages, but induction and maintenance of lysogeny also require other functions, such as a repressor of productive infection. It is possible that D29 has evolved from a temperate phage and become defective in one or more functions required for establishment of lysogeny. Alternatively, DNA integration may be an intermediate step in the phage replication cycle.

\section{ACKNOWLEDGEMENTS}

Miguel Viveiros is a recipient of a doctoral fellowship from Junta Nacional de Investigação Científica e Tecnológica, Portugal. We thank Nicholas Price for proof-reading the manuscript.

\section{REFERENCES}

Barletta, R. G., Kim, D. D., Snapper, S. B., Bloom, B. R. \& Jacobs, W. R., Jr (1992). Identification of expression signals of the mycobacteriophages Bxb1, L1 and TM4 using the Escherichia-Mycobacterium shuttle plasmids pYUB75 and pYUB76 designed to create translational fusions to the lacZ gene. J Gen Microbiol 138, 23-30.

Bowman, B. U. (1958). Quantitative studies on some mycobacterial phage-host systems. J Bacteriol 76, 52-62.
David, M., Lubinsky-Mink, S., Ben-Zvi, A., Ulitzur, S., Kuhn, J. \& Suissa, M. (1992). A stable Escherichia coli-Mycobacterium smegmatis plasmid shuttle vector containing the mycobacteriophage D29 origin. Plasmid 28, 267-271.

Devereux, J., Haeberli, P. \& Smithies, O. (1984). A comprehensive set of sequence analysis programs for the VAX. Nucleic Acids Res 12, 387-395.

Donnely-Wu, M. K., Jacobs, W. R., Jr \& Hatfull, G. F. (1993). Superinfection immunity of mycobacteriophage L5: applications for genetic transformation of mycobacteria. Mol Microbiol 7, $407-417$.

Froman, S., Will, D. W. \& Bogen, E. (1954). Bacteriophage active against virulent Mycobacterium tuberculosis. I. Isolation and activity. Am J Public Health 44, 1326-1333.

Hall, D. H. \& Tessman, I. (1966). T4 mutants unable to induce deoxycytidylate deaminase activity. Virology 29, 339-345.

Hatfull, G. F. \& Sarkis, G. J. (1993). DNA sequence, structure and gene expression of mycobacteriophage L5: a phage system for mycobacterial genetics. Mol Microbiol 7, 395-405.

Houssaini-Iraqui, M., Lazraq, R., Clavel-Sérès, S., Rastogi, N. \& David, H. L. (1992). Cloning and expression of Mycobacterium aurum carotenogenesis genes in Mycobacterium smegmatis. FEMS Microbiol Lett 90, 239-244.

Kempsell, K. E., Ji, Y. E., Estrada, I. C., Colston, M. J. \& Cox, R. A. (1992). The nucleotide sequence of the promoter, $16 \mathrm{~S}$ rRNA and spacer region of the ribosomal RNA operon of Mycobacterium tuberculosis and comparison with Mycobacterium leprae precursor rRNA. J Gen Microbiol 138, 1717-1727.

Labidi, A., David, H. L. \& Roulland-Dussoix, D. (1985). Restriction endonuclease mapping and cloning of Mycobacterium fortuitum var. fortuitum plasmid pAL5000. Ann Inst Pasteur Microbiol 136B, 209-215.

Lazraq, R., Clavel-Sérès, S., David, H. L. \& Roulland-Dussoix, D. (1990). Conjugative transfer of a shuttle plasmid for Escherichia coli to Mycobacterium smegmatis. FEMS Microbiol Lett 69, 135-138.

Lazraq, R., Houssaini-Iraqui, M., Clavel-Sérès, S. \& David, H. L. (1991). Cloning and expression of the origin of replication of mycobacteriophage D29 in Mycobacterium smegmatis. FEMS Microbiol Lett 80, 117-120.

Lee, M. H. \& Hatfull, G. F. (1993). Mycobacteriophage L5 integrase-mediated site-specific integration in vitro. $J$ Bacteriol 175, 6836-6841.

Lee, M. H., Pascopella, L., Jacobs, W. R., Jr \& Hatfull, G. F. (1991). Site-specific integration of mycobacteriophage L5: integrationproficient vectors for Mycobacterium smegmatis, Mycobacterium tuberculosis, and bacille Calmette-Guérin. Proc Natl Acad Sci USA 88, 3111-3115.

Maley, G. F., Guarino, D. U. \& Maley, F. (1983). Complete amino acid sequence of an allosteric enzyme, T2 bacteriophage deoxycytidylate deaminase. J Biol Chem 258, 8290-8297.

Mizuguchi, Y. (1984). Mycobacteriophages. In The Mycobacteria : a Source Book, pp. 641-662. Edited by G. P. Kubica \& L. G. Wayne. New York: Marcel Dekker.

Pefia, C. E. A., Stoner, J. E. \& Hatfull, G. F. (1996). Positions of strand exchange in mycobacteriophage L5 integration and characterization of the attB site. J Bacteriol 178, 5533-5536.

Peña, C. E. A., Lee, M. H., Pedulla, M. L. \& Hatfull, G. F. (1997). Characterization of the mycobacteriophage L5 attachment site, attP. J Mol Biol 266, 76-92.

Redmond, W. B. \& Ward, D. M. (1966). Media and methods for phage-typing mycobacteria. Bull WHO 35, 563-568. 
Sambrook, J., Fritsch, E. F. \& Maniatis, T. (1989). Molecular Cloning: a Laboratory Manual, 2nd edn. Cold Spring Harbor, NY : Cold Spring Harbor Laboratory.

Sanger, F., Nicklen, S. \& Coulson, A. R. (1977). DNA sequencing with chain-terminating inhibitors. Proc Natl Acad Sci USA 74, 5463-5467.

Snapper, S. B., Lugosi, L., Jekkel, A., Melton, R. E., Kieser, T., Bloom, B. R. \& Jacobs, W. R., Jr (1988). Lysogeny and transformation in mycobacteria: stable expression of foreign genes. Proc Natl Acad Sci USA 85, 6987-6991.

Snapper, S., Melton, R., Keiser, T. \& Jacobs, W. R., Jr (1990). Isolation and characterization of efficient plasmid transformation mutants of Mycobacterium smegmatis. Mol Microbiol 4, 1911-1919.
Snider, D. E., Jr, Raviglione, M. \& Kochi, A. (1994). Global burden of tuberculosis. In Tuberculosis: Pathogenesis, Protection, and Control, pp. 3-11. Edited by B. R. Bloom. Washington, DC: American Society for Microbiology.

Stover, C. K., de la Cruz, V. F., Fuerst, T. R., Burlein, J. E., Benson, L. A., Bennett, L. T., Bansal, G. P., Young, J. F., Lee, M. H., Hatfull, G. F., Snapper, S. B., Barletta, R. G., Jacobs, W. R., Jr \& Bloom, B. R. (1991). New use of BCG for recombinant vaccines. Nature 351, 456-460.

White, A., Foster, F. \& Lyon, L. (1962). Alteration in colony morphology of mycobacteria associated with lysogeny. J Bacteriol 84, 815-818.

Received 27 January 1997; revised 15 April 1997; accepted 22 April 1997. 